\title{
Research on the Online Spread of Rumors about the Quality and Safety of Agricultural Products in the New Media Era Using an Improved SEIRS Model
}

\author{
Qunxiang Zhang, ${ }^{1,2}$ Huilan Fang $\mathbb{D}^{3},{ }^{3}$ Peng Yao, ${ }^{1}$ and Haibin Zhang ${ }^{1}$ \\ ${ }^{1}$ School of Economics and Management, Zhejiang Agriculture and Forestry University, Hangzhou 311300, China \\ ${ }^{2}$ Zhejiang Institute of Rural Revitalization, Hangzhou 311300, China \\ ${ }^{3}$ College of Science, Zhejiang Agriculture and Forestry University, Hangzhou 311300, China
}

Correspondence should be addressed to Huilan Fang; fang_hl@163.com

Received 2 February 2021; Revised 16 July 2021; Accepted 4 August 2021; Published 11 August 2021

Academic Editor: Luca Pancioni

Copyright (c) 2021 Qunxiang Zhang et al. This is an open access article distributed under the Creative Commons Attribution License, which permits unrestricted use, distribution, and reproduction in any medium, provided the original work is properly cited.

\begin{abstract}
The rapid development and dissemination of new media have increased online rumor transmission. Fermentation of some rumors regarding quality and safety of agricultural products has created serious problems that have negatively impacted agriculture in China and the consumption of goods such as poultry. Thus, it is imperative to explore the network dissemination mechanisms regarding how rumors about agricultural product quality and safety spread in the new media era. This study used "fast-growing chicken hormone-boosting" as the base rumor, and it developed an improved SEIRS model to identify the spread of rumors regarding agricultural product quality and safety. This study innovatively introduced a conversion rate and analyzed its impact on the secondary transmission of rumors. Using MATLAB software simulation, the research produced the following findings: an increase in netizens does not affect the threshold of rumor outbreaks; improving the immunity rate of contact netizens and the cure rate of spread netizens is the key to both controlling Internet ru 'mors and reducing the contact rate of ordinary netizens. At the same time, an increase in the conversion rate could lead to a secondary outbreak of rumors.
\end{abstract}

\section{Introduction}

China's Internet users were projected to reach 904 million as of March 2020, with a penetration rate of $64.5 \%$, according to the 45th edition of the China Internet Development Statistics Report released by the China Internet Network Information Center (CNNIC). Furthermore, the number of mobile phone users has increased by 79.92 million since the end of 2018, and $99.3 \%$ of Chinese people have mobile Internet access [1]. The rapid development and influence of new media technology, including microblogs, TikTok, and instant messaging, are profoundly changing people's communication styles as well as the public opinion environment. In addition, people's attitudes, cognition, opinions, and emotions around social phenomena have increasingly become focal points of public opinion and sources of trends and influence. In recent years, rumors about agricultural product quality and safety have appeared from time to time. The Ministry of Agriculture of China has uncovered the top ten typical rumors regarding agricultural product quality and safety, including "fast-growing chickens raised with hormones." Some agricultural product-targeted rumors relating to quality and safety have evolved into serious events due to the decentralized, nonlinear, distorted, and rapid dissemination possible via new media. This has had a continuing negative impact on industry development and people's consumption. Therefore, the supervision and management of Internet rumors has become more urgent. In particular, researchers are paying close attention to the spread of rumors regarding issues of high concern among consumers: the quality and safety of agricultural products.

In recent years, the generation and evolution of Internet rumors in the new media environment have become hot topics for research. Scholars have conducted exploratory 
research around the modeling and simulation of rumors, including the following: the $D-K$ model, which is based on the classic SIR model [2]; the SIHR rumor spread model with an additional hibernator [3]; the SIRaRu model, which introduces the oblivion rate [4]; the SSIC Supernetwork Rumor Spreading Model, which considers an intervention mechanism [5]; the ISRC model, with the addition of a new control group [6]; and the SInQR model for the spread of rumors from multiple dissemination sources [7]. These studies have conducted modeling and simulation of the spread of rumors using different factors; thus, they have provided important references for the subsequent exploration of the spread and evolution of Internet rumors. However, the existing research shows that most models are constructed on abstracted reality and require improvements. In general, most research has been conducted from the perspective of a static network, ignoring the interaction and interference between netizens. Additionally, in the viral rumor spreading process, there is always a possibility of, essentially, reinfection of immune people due to the weakening of immunity. Besides, with the prosperity and development associated with new media, the netizen community has grown. Considering the above deficiencies and on the basis of relevant research and the real-world situation of Internet spread of rumors, this study proposes an improved SIR model for simulating the spread of agricultural product quality and safety rumors, helping to reveal detailed rules of Internet transmission and the evolution of agricultural product quality and safety rumors in the new media era. Moreover, this study provides suggestions for future theoretical discussion and practice for the academic community.

\section{Literature Review}

In 1927, Kermack and McKendrick [8] created the classic SIR model of epidemic transmission based on differential dynamics, in which $S$ (susceptible) represents the susceptible, $I$ (infective) refers to the infected, and $R$ (removed) represents the immune individuals. As the information dissemination mechanism of social networks and the spread of epidemic are highly similar, more scholars are expanding the use of the SIR model to explore the mechanisms of rumor dissemination. Based on the existing research, most of the studies focus on the improvement of the model and its expansion into other application areas. In terms of improving the model, Daley and Kendall [2] proposed the $D-K$ model, which is based on SIR. Maki and Thomson [9] revised the DK model and proposed the MK model. Zan et al. [10] then introduced the SICR (susceptible-infectivecounterattack-refractory) model to study the spread of rumors under different parameters. Dong et al. [11] proposed a new SEIR rumor dissemination model to analyze the inherent characteristics of the rumor spread process in social networks. Zhu et al. [12] analyzed the local stability, global stability, and optimal control of the SEIR rumor spreading model based on Dong et al. [11], and they proposed a modified rumor spreading model to discuss its local and global stability. Zhang and Zhu [13] proposed the I2S2R model to explore the threshold of rumor dissemination and its global stability. Jia et al. [14] proposed an improved SIR model and established the corresponding mean-field equation that distinguished two propagation channels of rumor spreading on social networks: point to point propagation and group propagation. Wang et al. [15] viewed rumor spreading within the multilingual environment, further establishing a new model with a cross-transmitted mechanism. Huo and Chen [16] proposed a stochastic rumor spreading model that considered the existence of time delay and the disturbance of white noise with Holling II functional response function.

Furthermore, Qiu et al. [17] put forward a new SIR rumor dissemination model (SIR-IM) with an influence mechanism (IM) to incorporate the number of communicators into the probability of dissemination. Zhang and $\mathrm{Li}$ [18] investigated the specific rumor propagation of Monte Carlo simulations when the spreading rate is small. Generally speaking, improvement of the SIR model is the main avenue of exploration in the study of rumor spread.

Another area relevant to model improvement has involved research on using complex networks, such as smallworld and scale-free networks, due to the topological features of social networks and their large scale. Zanette [19] first developed a rumor dissemination model by using a small-world network and found the critical threshold value for rumor spreading in a uniform network. Moreno et al. [20] studied the MK model on a scale-free network and found that the scale of rumor spread was influenced by the network structure and the speed with which rumors spread. Meanwhile, Nekovee et al. [21] explored the gossip propagation state in a network with regular complexity by combining the MK model with the SIR model. Zhu et al. [22] produced a comprehensive investigation of the influence of forced silence functions, time delay, and network topology on rumor propagation in online social networks. Considering network supervision, a novel delayed SIR epidemiclike rumor propagation model in both homogeneous and heterogeneous networks has been proposed. Yan et al. [23] studied a rumor spreading model in the homogeneous network called 2SIH2R. They introduced a discernible mechanism and a confrontation mechanism to quantify the level of people's cognitive abilities and the competition between rumor and truth. Based on the role of macroview mechanisms in the spread of rumors, Lü et al. [24] found that information was more easily disseminated in smallworld networks by modeling integrated memory effects and nonredundant contact factors. Wang and Wang [25] discussed the spread of rumors in uniform and nonuniform networks by using the average field theory. They found that the existence of network media greatly reduces the critical threshold and expands the final scope of rumor spread. In addition, Zhu and Wang [26] showed that, at the same noise intensity, rumors spread on the Barabási-Albert (BA) network much faster than on the Watts-Strogatz (WS) network. The introduction of complex networks provides a useful complement to the study of rumor spread in large-scale, open, nonuniform social networks. Zhang and Qiu [27] introduced the unknown person suspicion mechanism to 
establish the SIR-CM rumor propagation model. Based on the mean field theory, the spreading process of rumor in WS network and BA network was expounded.

With the rapid growth of mobile Internet, the spread of rumors on mobile social networks has become a new field of research in expanding applications. Based on the SIR model, Zhao et al. [28] developed a model of rumor dissemination on Live Journal (an online social blog platform), and the research showed that the forgetting mechanism has a significant impact on the spread of rumors on social networks. Afassinou [29] presented a SEIR model with a latent state, finding that increased education among people decreased their spreading of rumors. Liang and Wan [30] set up an ISMR rumor dissemination model to analyze the impact of media clarification on rumor dissemination and found that the more authoritative the media and the earlier the intervention are, the more rapidly the rumors could be extinguished. At the same time, Jiang and Yan [31] established a new SIR rumor dissemination model for online social networks (OSNs) using nodes to describe the dynamics of the number of rumor spreaders, and they found that when the basic reproduction number is less than 1 , the rumor will disappear. Liu et al. [32] built a URBD model to explore the spread of social network rumors, demonstrating the partial and holistic stability of rumor spread based on delayed reading by social network users. Dong et al. [33] set up a rumor spreading model in online social networks based on the European fox rabies SIR model that considers the impact of changing the number of online social network users and combines the transmission dynamics to set up a population dynamics model of rumor spreading in online social networks. Based on the classic SIR model, Gu et al. [34] added influencing factors such as the number of mobile social network users for optimization. Then, combining this with the theory of communication dynamics, they established a dynamic evolution model of participants in rumor propagation on a mobile social network. Based primarily on the cholera model, Amine et al. [35] proposed a new version of an existing IRCSS model to take into account the expert pages specializing in rumor dissemination, and they recommend an optimal control strategy to combat the rumor spreading.

In summary, there has been progress in the academic community in research into the spread of Internet rumors, and most of it is based on the SIR model with application, revision, expansion, and integration of tools such as complex networks to enable the model to simulate a real network environment more accurately. However, due to the repetitiveness of the spread of rumors and the continuous development of new media, the SIR model must be constantly improved to mimic the reality of the dissemination process. This study therefore proposed the SEIRS network dissemination model to examine the dissemination of rumors about agricultural product quality and safety. The model not only integrates the characteristics of increasing media users in the new media era but also innovatively examines from a dynamic perspective how the reinfection of immune people affects the secondary spread of rumors. The study used MATLAB software for the simulation analysis to explore the mechanism of network dissemination and evolution of rumors about agricultural product quality and safety to suggest countermeasures that might be taken for the management of Internet rumors.

The remainder of the paper is organized as follows. Section 3 presents the model hypotheses and builds the SEIRS model. Section 4 analyzes model results and presents simulation analysis. Section 5 concludes the paper.

\section{Methods and Materials: Building the SEIRS Model}

3.1. Model Hypothesis. With the rapid development and penetration of new media, the total group of netizens $(N)$ continues to increase, and the growth rate in their number in the new media era is set as $K$. Due to the openness of the Internet, netizens can come into contact with many kinds of rumors related to the quality and safety of agricultural products, presenting different dissemination states, which can be interchangeable. The netizens concerned with these issues are categorized as ordinary netizens $(S)$, who have no access to rumors and who either do not disseminate or are immune to such information; contact netizens $(E)$ who browse online rumors and have not decided whether to spread them and who may be converted to spread netizens or immune netizens; spread netizens (I), who come into contact with rumors and spread them; immune netizens $(R)$, who come into contact with rumors and neither spread them nor become infected, but who can have weakened immunity later upon the secondary spread of rumors and may then be converted to ordinary netizens and reinfected by rumors.

3.2. Model Building. For a given $t$ moment, the density ratios of ordinary netizens, contact netizens, spread netizens, and immune netizens to the total are set as $S(t), E(t), I(t)$, and $R$ $(t)$; the status transfer relationships between the four categories of netizens are shown in Figure 1. According to the SIR naming rules for epidemic, the online spread model of rumors about agricultural product quality and safety is the improved SEIRS model.

Based on the idea of system dynamics modeling, the following SEIRS differential system dynamic model can be built, and the main parameters and explanations are shown in Table 1.

$$
\begin{aligned}
& \frac{\mathrm{d} S}{\mathrm{~d} t}=k S-\alpha S I+\beta R, \\
& \frac{\mathrm{d} E}{\mathrm{~d} t}=\alpha S I-\lambda E-\mu E, \\
& \frac{\mathrm{d} I}{\mathrm{~d} t}=\lambda E-\gamma I, \\
& \frac{\mathrm{d} R}{\mathrm{~d} t}=\mu E+\gamma I-\beta R .
\end{aligned}
$$

Among them, $k$ is the increase rate of an average netizen over time; $\alpha$ represents the probability of ordinary netizens coming into contact with rumor information; $\lambda$ and $\mu$ stand 


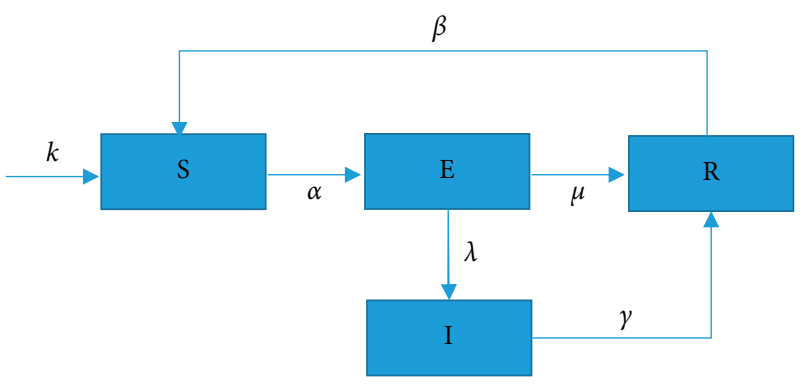

FIgURE 1: The improved SEIRS model.

TABLE 1: The main parameters of the SEIRS model and their explanations.

\begin{tabular}{lcr}
\hline Parameters & Definition & Explanation \\
\hline$k$ & $\begin{array}{c}\text { The growth rate of new } \\
\text { netizens }\end{array}$ & The rate of growth in Internet users over time in the new media era \\
$\alpha$ & Contact rate & The probability of ordinary netizens getting exposed to rumors about agricultural product \\
$\lambda$ & Infection rate & The probability of netizens exposed to rumors converting to spread netizens \\
$\mu$ & Immunity rate & The probability of exposed netizens converting to immune netizens without spreading rumors \\
$\gamma$ & Cure rate & The probability of spread netizens converting to immune netizens \\
$\beta$ & Conversion rate & The probability of immune netizens converting to ordinary netizens
\end{tabular}

for the spread rate and immunity rate of the Internet users exposed to rumors; $\gamma$ is the rate of immunity of spread netizens; and $\beta$ represents the rate of immune netizens who convert into ordinary netizens.

\section{Results}

To analyze the stability of the model, we considered the stable points within the region of $\{(S, E, I, R) \mid S \geq 0, E \geq 0$, $I \geq 0, R \geq 0$, 且 $S+E+I+R=1\}$.

When $S=E=I=R=0$, the model can be seen as reaching equilibrium, and $(0,0,0,0)$ can be called the zero equilibrium point at which the total number of infectious Internet users is 0 . If the whole rumor has no source of infection, it does not break out.

Set $\left(S^{*}, E^{*}, I^{*}, R^{*}\right)$ as a nonzero equilibrium point, and the results are calculated. Set the right side of the model to zero.

$$
\begin{aligned}
& \text { Based on } \lambda E-\gamma I=0, E^{*}=(r / \lambda) I^{*} \text {. } \\
& \text { Based on } \mu E+\gamma I-\beta R=0, R^{*}=(1+(\mu / \lambda))(r / \beta) I^{*} \text {. } \\
& \text { Based on } \alpha S I-\lambda E-\mu E=0, S^{*}=(1+(\mu / \lambda))(r / \alpha) . \\
& \text { Based on } S+E+I+R=1, I^{*}=\left(1-K_{0} /(1+(r / \lambda)+\right. \\
& \left.\left.K_{0}(\alpha / \beta)\right)\right) \quad \text { can be concluded, in which } \\
& K_{0}=((\lambda+\mu) / \lambda)(r / \alpha) \text {. At this point, if a rumor spreads } \\
& \text { without containment, it may break out. }
\end{aligned}
$$

When $K_{0} \geq 1$, then $I^{*} \leq 0$ in this model. Thus, there is no nonzero equilibrium point, but only a zero equilibrium point, and the rumor dissipates over time and does not require research.

When $0<K_{0}<1, I^{*}>0$. There is a nonzero equilibrium point $\left(S^{*}, E^{*}, I^{*}, R^{*}\right)$. Over time, without the corresponding intervention, rumors can erupt.
Set $K_{0}$ as the threshold for model stability. From the perspective of controlling the spread of rumors, it has been found that the larger $K_{0}$ is, the more conducive it is to controlling the spread of rumors. With $K_{0}=(\lambda+\mu / \lambda)(r / \alpha)$, it has been found that $K_{0}$ has no relation to $k$, the new netizen coefficient, indicating that the increase in the number of netizens will not affect the rumor outbreak threshold.

Then, $\mu$ is the immunity rate of exposed netizens, and the increase in $\mu$ can effectively improve $K_{0}$. In actual management, $\mu$ can be increased by improvements in scientific literacy and knowledge popularization to enhance netizens' abilities to identify rumors. $\gamma$ represents the cure rate of spread netizens, and the increase in its value can also effectively improve $K_{0}$. In the process of actual supervision, it is possible to improve $K_{0}$ through the open and transparent release of authoritative information, expert refutation of rumors, and other methods. Then, $\alpha$ is the probability of regular netizens exposed to rumors, and decreasing it can effectively improve $K_{0}$. In the actual management process, it is necessary to control the spread of false information in a timely manner and to respond actively to the concerns of society.

4.1. Simulation Analysis. To further study the impact of different parameters on the spread of network rumors, this study employed MATLAB software to simulate the spread of rumors regarding agricultural product quality and safety in the new media era.

4.2. Setting of Experimental Parameters. The rumor "fastgrowing chicken boosted by hormones" is on the top 10 list of rumors about agricultural products that is published by 
China's Ministry of Agriculture. In the rumor, the "fastgrowing chicken" is characterized by large size, fast growth, and white feathers. This type of chicken matures in slightly over 40 days. According to traditional Chinese practice, a chicken requires at least a half of a year to mature, while the white-feathered chicken can be sold in just over 40 days. On the Internet, the animal was then labeled "fast-growing chicken," and the Internet rumor claimed that its rapid growth is due to being fed with hormones. This is the origin of the rumor of "fast-growing chicken boosted by hormones." This rumor has continued to spread on the Internet, and many consumers have worried that eating whitefeathered chicken may be harmful to their health, which has caused the sales of white-feathered chickens to plummet and the price to fall. The rumor has had serious consequences for the poultry industry and has attracted attention from relevant companies, netizens, and the government. This study uses the "fast-growing chicken" rumor for its simulation analysis.

In line with the needs of the simulation experiment, we set the initial parameters of the Internet spread model based on the rumor "fast-growing chickens boosted by hormones." In the early stage of the rumor's spread, few netizens saw it, and the initial values of the model were set as $S_{0}=0.95$, $E_{0}=0.03, I_{0}=0.02$, and $R_{0}=0.0$.

According to the China Internet Development Statistics Report of March 2020, the natural growth rate of new netizens was set as $k=0.0026$. For a comprehensive study of the influence of major parameters such as contact rate and immunity rate, this study set up 5 groups of 16 parameter schemes, as shown in Table 2. The corresponding simulation results are displayed in Figures 2-7.

4.3. Analysis of the Simulation Results. As shown in Figure 8, the trajectory of the four types of new media netizen changes can be observed from the experiment results over time. When rumors about the quality and safety of agricultural products began to spread, the $I(t)$ and $E(t)$ groups grew rapidly, reached their peaks, and then began to decline, finally stabilizing at $t=25$. The $S(t)$ group declined rapidly, and then the rate of decline slowed down and eventually stabilized.

\subsubsection{Analysis of the Impact of the Contact Rate on the Model} Results. Figure 2 shows the impact of the change of $\alpha$ on the model results, with $\alpha$ being the probability of Internet users coming into contact with rumors about agricultural product quality and safety. In Figure 2(a), with the decrease of $\alpha$, the infection rate of ordinary netizens $S(t)$ also slows down; in Figures 2(b) and 2(c), with the drop of $\alpha$, the increase in rate of infected netizens slows down in the early stage, but the peak eventually arrives, and the number of netizens infected when it comes to a stable state also reduces. This explains that reducing contact rate $\alpha$ can greatly contain the spread of rumors about agricultural product quality and safety.

Based on the above analysis, combined with the characteristics of the new media and the impact of all types of Internet rumors, netizens will gradually pay less attention to quality and safety rumors until new information is added to the rumors. Therefore, given that $\alpha$ can decrease over time, MATLAB was used to estimate the parameter values and set them at $\alpha=1.5-0.02 t$.

From Figure 9, we can see the effect of the change of $\alpha$ on the model results. With the increase in time, the contact rate of $\alpha$ is relatively larger between $t=1$ and $t=15$ : The average netizen $S(t)$ decreases rapidly, and both the spread netizen $I(t)$ and the immune netizen $R(t)$ increase, but during the late stage of $t>15$, when the contact rate is smaller, the overall number of spread netizens $I(t)$ decreases, and they convert to immune netizens $R(t)$. However, the number of immune netizens did not grow, and with the development of the event, immunity weakened among the immune netizens, and they gradually converted to ordinary netizens. This is reflected by the statistics: When $t>15, S(t)$ gradually increased, and until $t=80,83 \%$ of the ordinary netizens no longer paid attention to this rumor. Over time, the rumor dissipated, and the netizens all recovered to $S(t)$, until the relevant rumors about agricultural product quality and safety spread for the second time, when the four types of Internet users began to move to the next round of interchanges.

\subsubsection{Analysis of the Impact of the Natural Growth Rate of} Netizens on the Model Results. The parameter $k$ represents the natural growth rate of ordinary netizens. Here, we set the values separately at $k=0.002, k=0.005, k=0.01$, with the rest of the parameters unchanged, as seen in Figure 3. The change of $k$ in the model had little effect on the change trend of the population density of the four types of netizens in the model. Although the number of spread netizens changed slightly, this did not affect the overall evolution of the curve, which is consistent with the results of the previous threshold analysis. In other words, the change of $k$ does not affect the rumor outbreak threshold.

\subsubsection{Analysis of the Effect of Immune Rate on Model Results.} The parameter $\mu$ stands for the immune rate of netizens who have been exposed to rumors about agricultural product quality and safety. In this study, we set the values separately at $\mu=0.2, \mu=0.4, \mu=0.6$, with the remaining parameters unchanged, as seen in Figure 4. In Figure 4(a), the population density of ordinary netizens remains unchanged between $t=1$ and $t=5$; when $t>5$, the increase of $\mu$ can slow down the infection rate of ordinary netizens. Figures $4(\mathrm{~b})$ and 4(c) show that, with the increase of $\mu$, the early-stage growth rate of spread netizens can be slowed down, resulting in a late arrival peak, and the number of spread netizens reduces when it comes to the stable state. This demonstrates that the increase of $\mu$ can greatly control the spread of rumors about agricultural product quality and safety. Relevant governmental departments can thus guide netizens by releasing authoritative information about agricultural products' quality safety and enhance netizens' ability to identify false information, resulting in the improvement of the immunity rate of netizens to effectively control the spread speed of rumors. 
TABLE 2: Schemes for model parameter setting.

\begin{tabular}{|c|c|c|c|c|c|c|c|}
\hline Scheme & & $\beta$ & $\lambda$ & $\mu$ & $\gamma$ & $k$ & Notes \\
\hline \multirow{3}{*}{ Group 1} & 1 & 0.1 & 0.6 & 0.4 & 0.1 & 0.0026 & \multirow{3}{*}{ Set $\alpha$ in scheme 1 as 1.2 ; set $\alpha$ in scheme 2 as 0.9 ; set $\alpha$ in scheme 3 as 0.6} \\
\hline & 2 & 0.1 & 0.6 & 0.4 & 0.1 & 0.0026 & \\
\hline & 3 & 0.1 & 0.6 & 0.4 & 0.1 & 0.0026 & \\
\hline \multirow{3}{*}{ Group 2} & 4 & 0.1 & 0.6 & 0.4 & 0.1 & 0.002 & \multirow{3}{*}{ The value of $k$ changes, with $\alpha=1.5-0.02 t$} \\
\hline & 5 & 0.1 & 0.6 & 0.4 & 0.1 & 0.005 & \\
\hline & 6 & 0.1 & 0.6 & 0.4 & 0.1 & 0.01 & \\
\hline \multirow{3}{*}{ Group 3} & 7 & 0.1 & 0.6 & 0.2 & 0.1 & 0.0026 & \multirow{3}{*}{ The value of $\mu$ changes, with $\alpha=1.5-0.02 t$} \\
\hline & 8 & 0.1 & 0.6 & 0.4 & 0.1 & 0.0026 & \\
\hline & 9 & 0.1 & 0.6 & 0.6 & 0.1 & 0.0026 & \\
\hline \multirow{3}{*}{ Group 4} & 10 & 0.1 & 0.6 & 0.4 & 0.1 & 0.0026 & \multirow{3}{*}{ The value of $\gamma$ changes, with $\alpha=1.5-0.02 t$} \\
\hline & 11 & 0.1 & 0.6 & 0.4 & 0.3 & 0.0026 & \\
\hline & 12 & 0.1 & 0.6 & 0.4 & 0.5 & 0.0026 & \\
\hline \multirow{4}{*}{ Group 5} & 13 & 0 & 0.6 & 0.4 & 0.1 & 0.0026 & \multirow{4}{*}{ The value of $\beta$ changes, with $\alpha=1.5-0.02 t$} \\
\hline & 14 & 0.1 & 0.6 & 0.4 & 0.1 & 0.0026 & \\
\hline & 15 & 0.3 & 0.6 & 0.4 & 0.1 & 0.0026 & \\
\hline & 16 & 0.5 & 0.6 & 0.4 & 0.1 & 0.0026 & \\
\hline
\end{tabular}

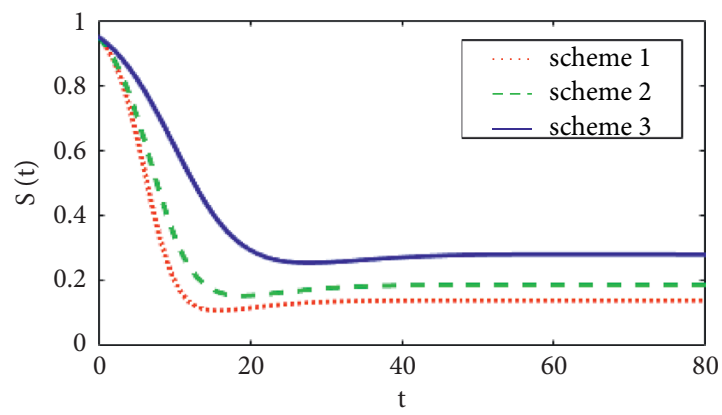

(a)

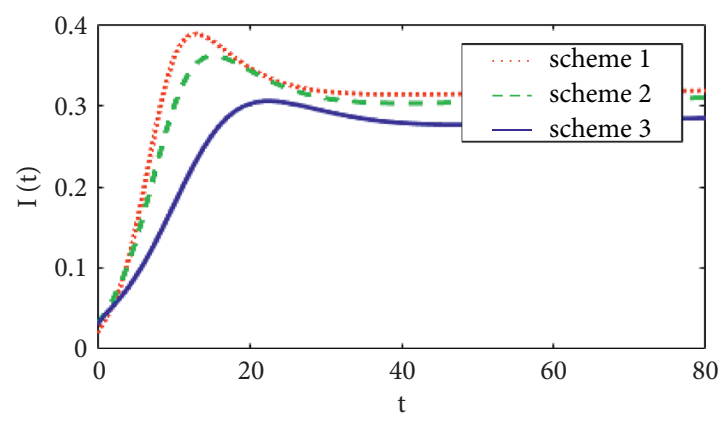

(c)

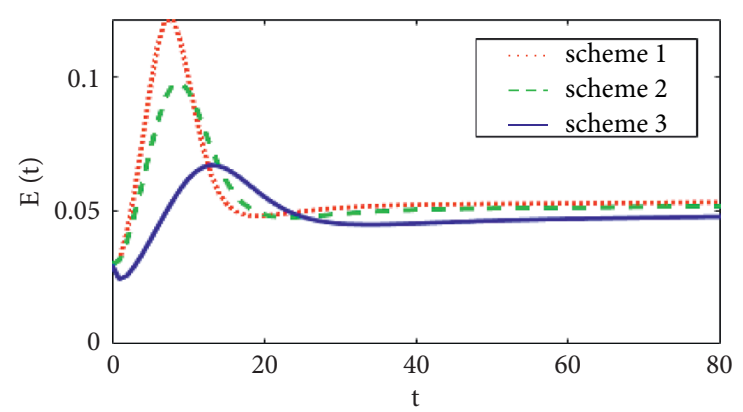

(b)

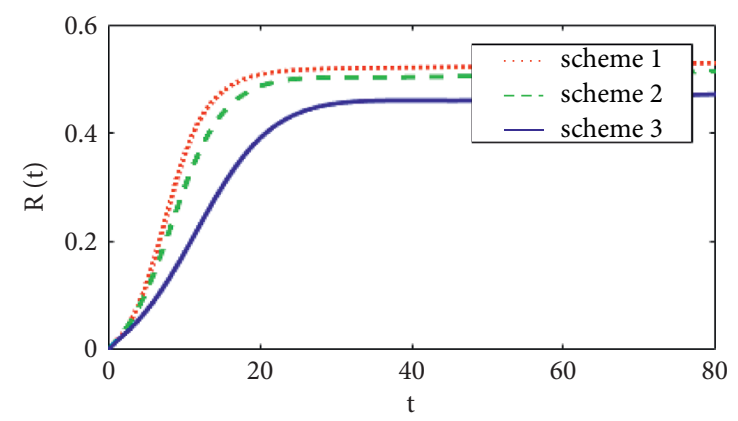

(d)

FIgURE 2: The curves of changes in the population density of netizens in the four categories at different contact rates of $\alpha$.

4.3.4. Analysis of the Effect of Cure Rate on Model Results. The parameter $\gamma$ is the cure rate of the spread netizens. Set $\gamma=0.1, \gamma=0.3, \gamma=0.5$ separately, with the remaining parameters unchanged, and the results of the model are shown in Figure 5, from which we can observe the impact of the changes of $\gamma$. Based on Figure 5(a), the increase of $\gamma$ can slow down the infection rate of average netizens. Based on Figures 5(b) and 5(c), the early-stage growth rate of spread netizens slowed down, with its peak arriving late, and the number of spread netizens reduced when it came to a stable state, even dropping to 0 when the value of $\gamma$ was relatively large. At this point, the rumor stopped spreading and discontinued its eruption. This demonstrates that increasing the cure rate is a direct way to effectively control the spread of agricultural product quality and safety rumors.

4.3.5. Analysis of the Impact of the Conversion Rate of $\beta$ on Model Results. Parameter $\beta$ represents the probability of immune netizens converting to ordinary netizens. Over time, the immunity of immune netizens weakens, resulting in some of them converting to ordinary netizens and getting reinfected. In other words, there is a possibility of secondary infection. Firstly, we set $\beta=0, \beta=0.1$, and the remaining 


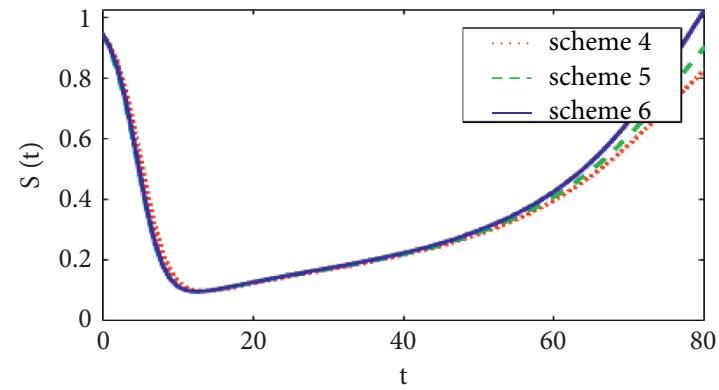

(a)

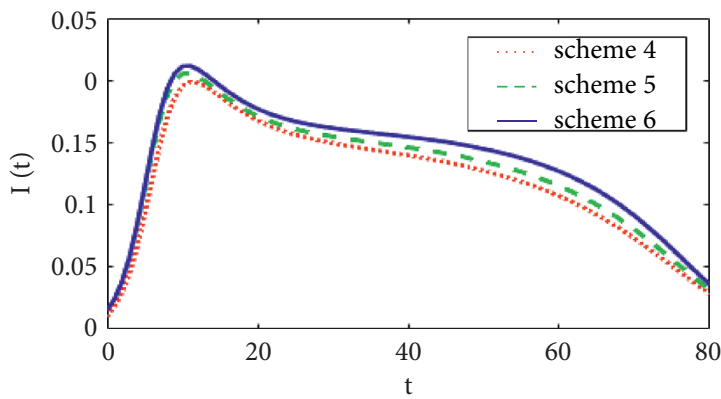

(c)

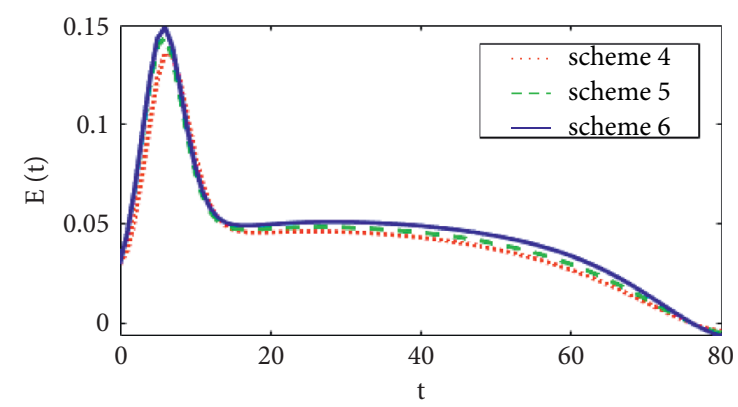

(b)

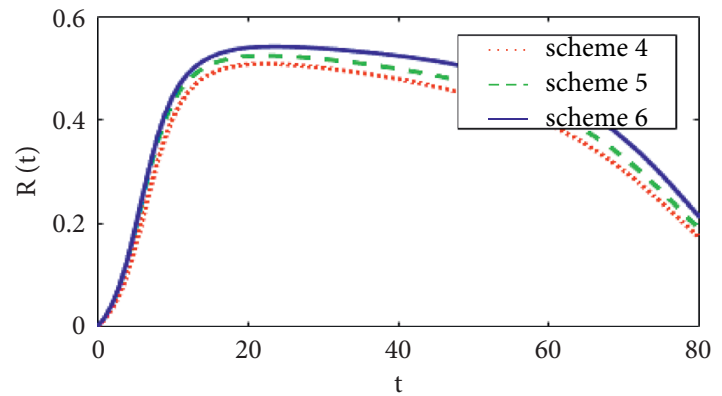

(d)

FIgURE 3: Curves of changes in the population density of netizens in the four categories at different values of $k$.

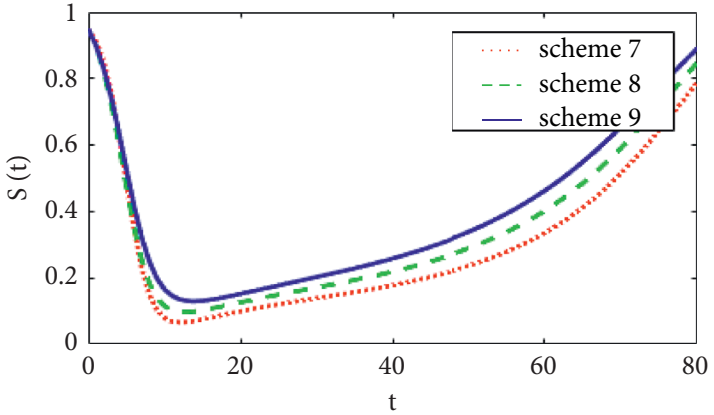

(a)

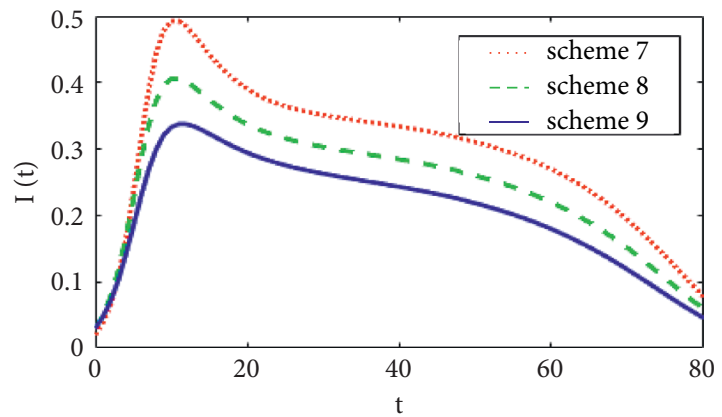

(c)

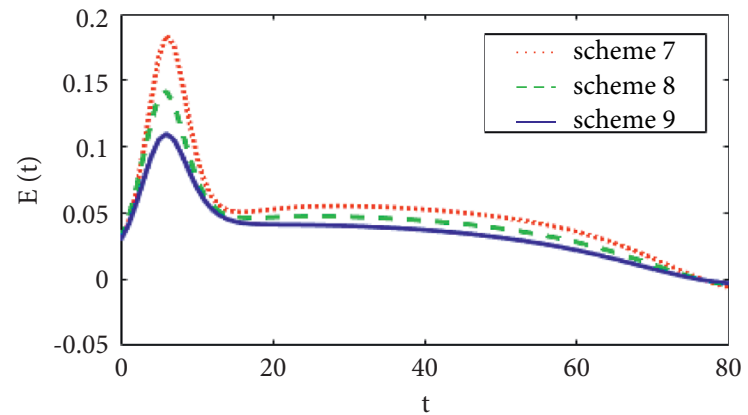

(b)

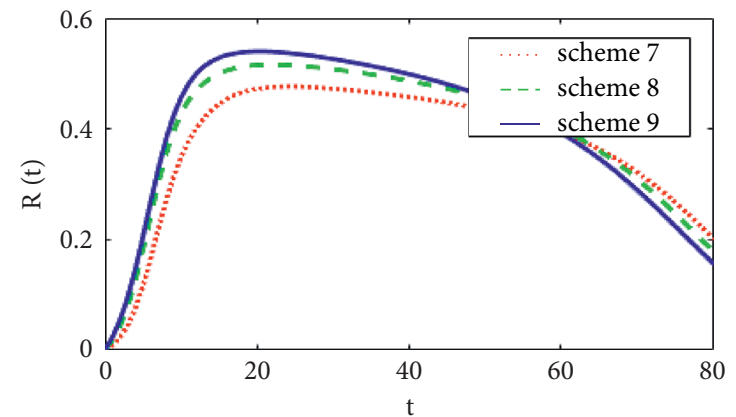

(d)

FIgURE 4: Curves of changes in the population density of the four categories of netizens at different values of $\mu$.

parameters stay unchanged. When $t=80$, the secondary diffusion of rumor information comes up. We can see from Figure 6 that if $\beta=0$, during the first spread of rumors, the spread netizens all convert to immune netizens and eventually tend to 0 . When the rumors spread for the second time, netizens all have immunity and are no longer affected by the rumors. However, in fact, when $\beta=0.1$, the immunity of immune netizens can 


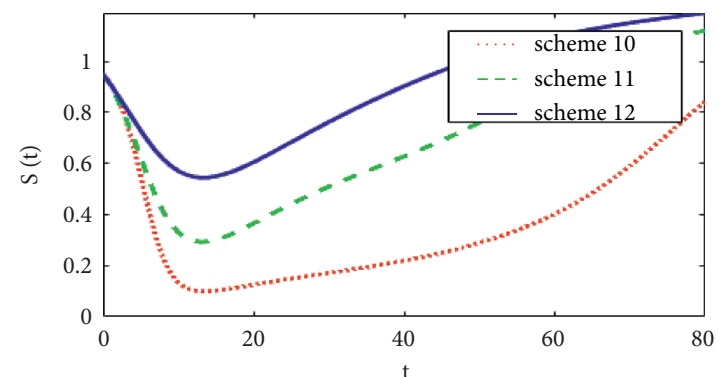

(a)

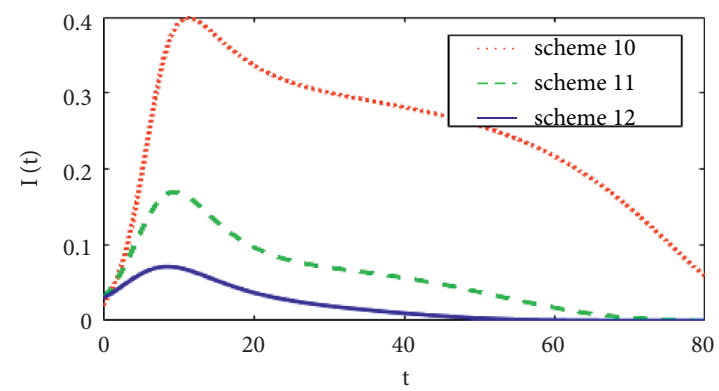

(c)

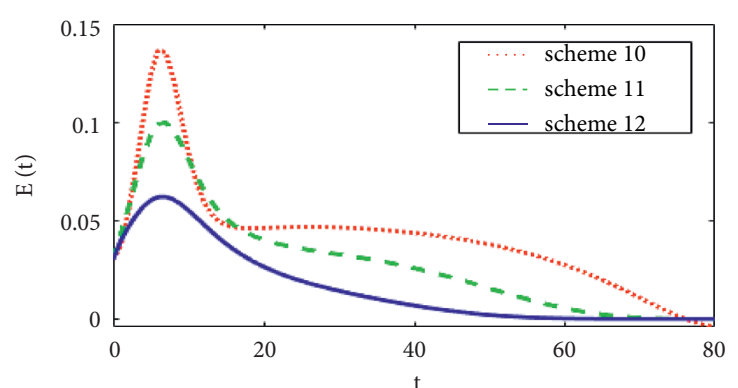

(b)

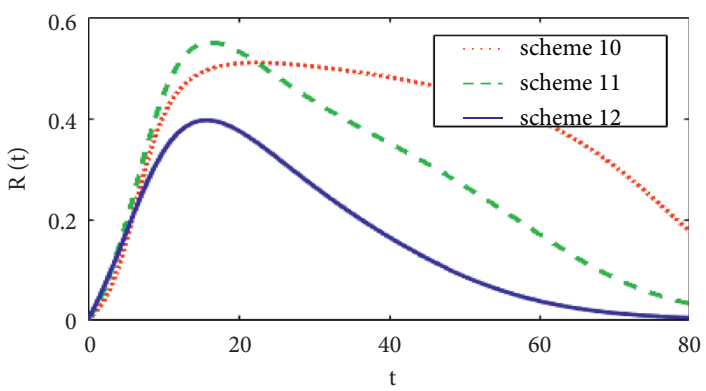

(d)

FIGURE 5: Curves of changes in the population density of the four categories of netizens at different values of $\gamma$.

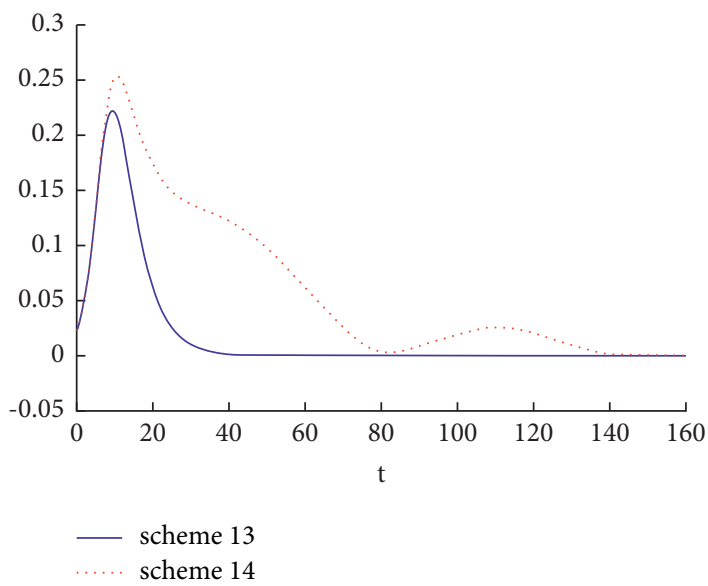

FIgURE 6: The impact of the conversion rate of $\beta$ on the population density of spread netizens.

weaken. Figure 6 shows that when $t=80$, the population density of spread netizens increases, and secondary rumor spread then starts.

Set $\beta=0.1, \beta=0.3, \beta=0.5$ separately, with the remaining parameters unchanged; the resulting model is shown in Figure 7. In Figure 7(a), with the increase of $\beta$, the increase in ordinary netizens slows down, although there is no obvious impact. In Figures 7 (b) and $7(\mathrm{c})$, with the increase of $\beta$ and with $t=10$, the population density of people at different stages of the spread netizens was increased, and the peak was slower to arrive, but the final spread of netizens in its steady state increased significantly. In Figure 7 (d), with the increase in the conversion rate of $\beta$, there is a significant decrease in the number of immune netizens. This shows that the probability of immune netizens converting to ordinary netizens increases; namely, they become reinfected and increase the population density of spread netizens. The increase in the number of spread netizens can lead to renewed discussions, and the government needs to quickly cut off the source of infection in the process of rumor spreading to prevent secondary spread.

Figure 10 shows the analysis of the change in the population density curve of the spread netizens at different diffusion times, when a rumor goes into secondary spread. The figure shows that the later the secondary diffusion, the smaller the growth of spread netizens, the lower the secondary peak, and the smaller the spread of the rumor event. Therefore, in the early stage of the outbreak of rumors, authoritative information should be quickly released in an 


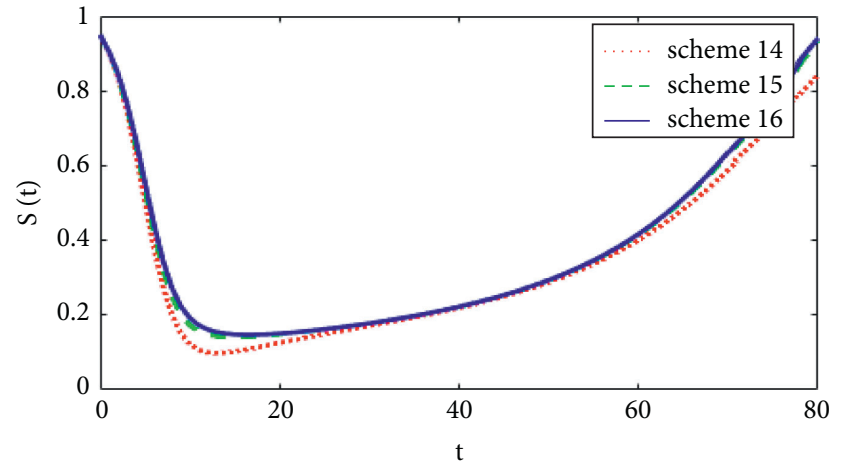

(a)

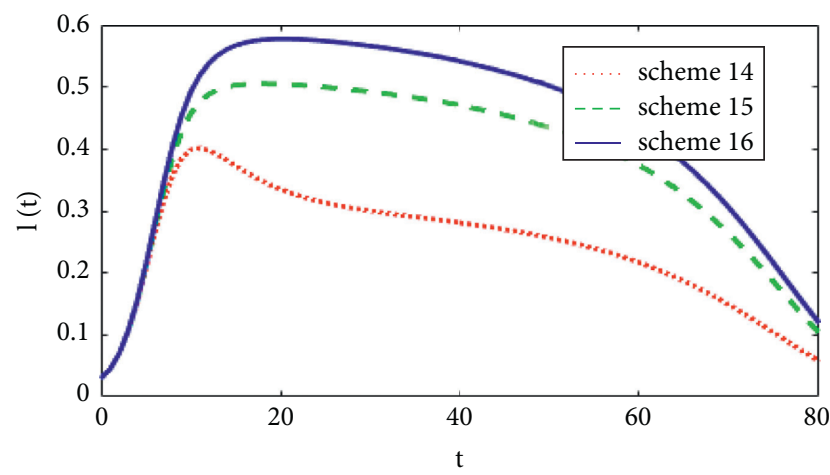

(c)

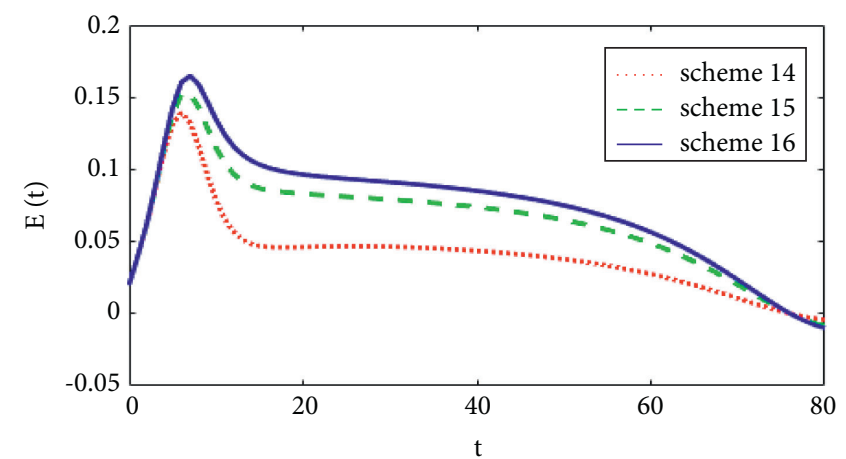

(b)

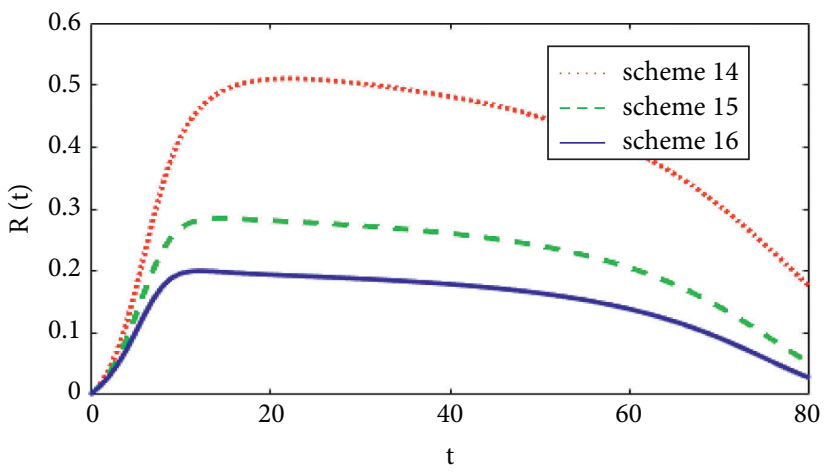

(d)

Figure 7: Changes in the population density curve of the four categories of netizens at different values of $\beta$.

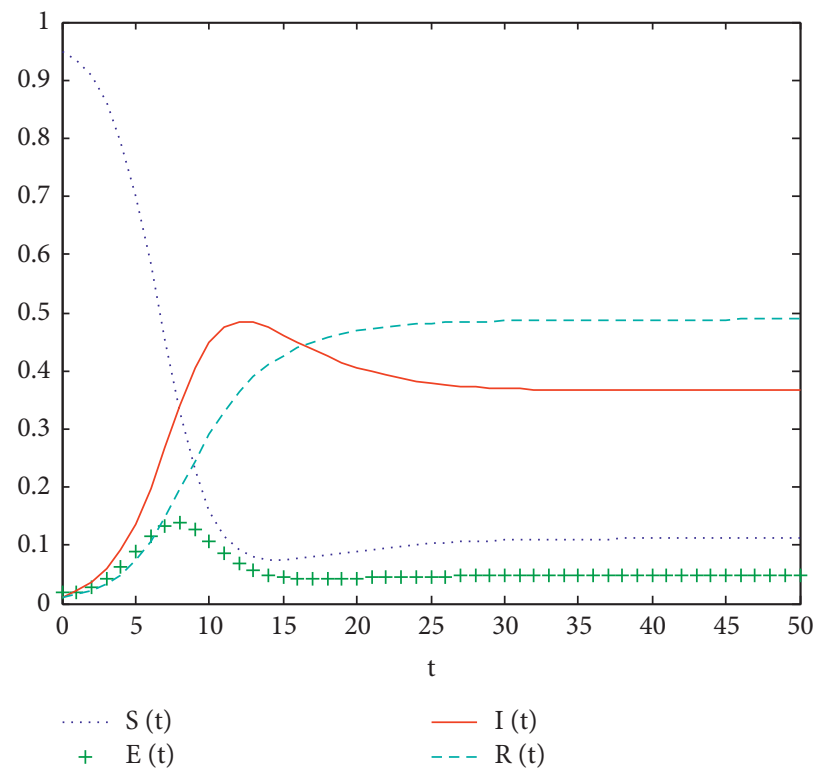

Figure 8: The population density curve of netizens in four categories at the initial parameter. 


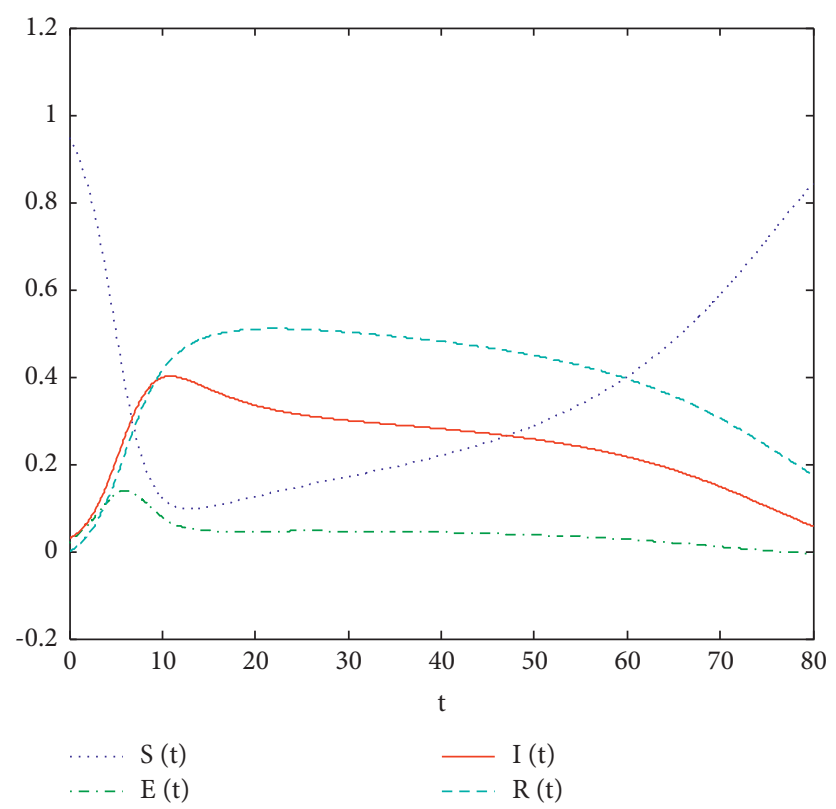

FIGURE 9: The population density curve of netizens in the four categories with parameter $\alpha=1.5-0.02 t$.

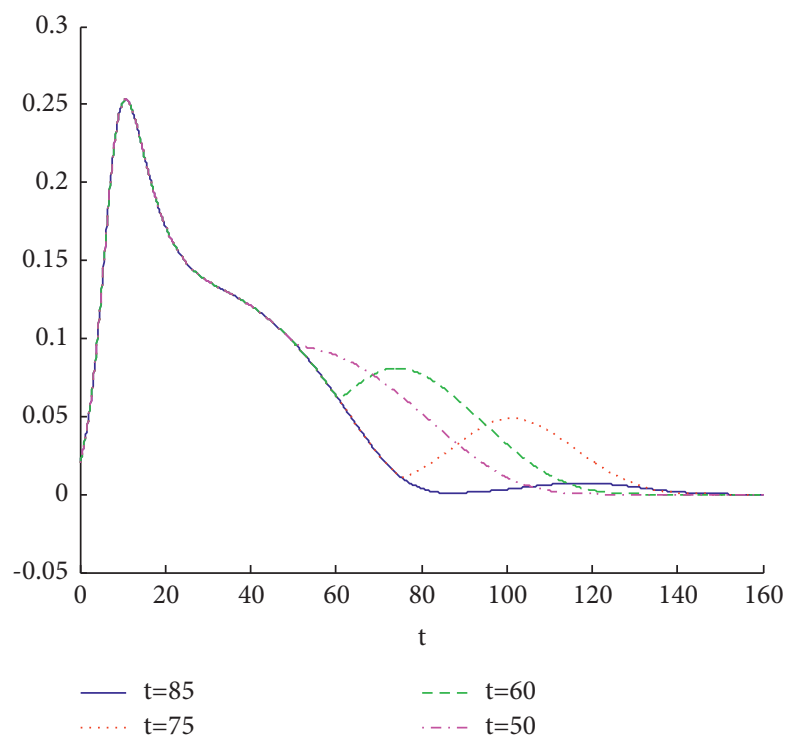

FIgURE 10: The change in the population density curve of the spread netizens at different secondary diffusion times.

open and transparent manner to reduce the possibility of secondary proliferation.

\section{Conclusions}

This study analyzed the spread of rumors about agricultural product quality and safety in the new media era, the trend of increasing numbers of netizens, and the possibility of mutual transfer between four categories of netizens. This research has taken the lead in constructing an improved SEIRS model by introducing the conversion rate. Based on the balance points and stability analysis of the model, the MATLAB simulation has revealed the mechanisms of spreading rumors about agricultural product quality and safety on new media.
Therefore, an innovative analysis of the effect of the conversion rate of $\beta$ on the secondary spread of rumors was conducted. The results show that the model stability threshold of $K_{0}$ has no relation to $k$, the coefficient of increasing numbers of new netizens, which indicates that the increase of a certain percentage of netizens will not directly affect the threshold for rumor outbreak. At the same time, improving the immunity rate of contact netizens of $\mu$ and the cure rate of spread netizens of $\gamma$ and reducing the contact rate of ordinary netizens of $\alpha$ can effectively control the spread of rumors. In addition, the increase in the conversion rate of $\beta$ can lead to a secondary outbreak of rumors, and the entire process of rumor dissemination needs to be monitored to prevent the repeated spread of rumors. 
As this study is based on a simulation experiment, the parameter values in the model are simulated data. Although in general the values can reflect the processes and mechanism of the spread of agricultural product quality and safety rumors in the new media era, inevitably, simulations' results may reflect some differences from real-world data. In view of this, there is a need to use real data related to agricultural product quality and safety by conducting a field study on the dissemination of Internet rumors and providing references and advice for managing those rumors.

\section{Data Availability}

The data used to support the findings of this study are included within the article.

\section{Conflicts of Interest}

The authors declare that there are no conflicts of interest regarding the publication of this study.

\section{Acknowledgments}

The authors would like to thank the National Social Science Fund of China for financially supporting this research under Grant no. 19BGL179.

\section{References}

[1] China Internet Network Information Center (CNNIC), "Statistical report on internet development in China," 2020, https://www.gov.cn/xinwen/2020-04/28/content_5506903.htm.

[2] D. J. Daley and D. G. Kendall, "Epidemics and rumors," Nature, vol. 204, no. 4963, p. 1118, 1964.

[3] L. Zhao, J. Wang, Y. Chen, Q. Wang, J. Cheng, and H. Cui, "SIHR rumor spreading model in social networks," Physica A: Statistical Mechanics and its Applications, vol. 391, no. 7, pp. 2444-2453, 2012.

[4] J. Wang, L. Zhao, and R. Huang, "SIRaRu rumor spreading model in complex networks," Physica A: Statistical Mechanics and its Applications, vol. 398, pp. 43-55, 2014.

[5] X. Liu, T. Li, and M. Tian, "Rumor spreading of a SEIR model in complex social networks with hesitating mechanism," Advances in Difference Equations, vol. 2018, p. 391, 2018.

[6] H. A. Ebadizadeh and H. Haghbayan, "Dynamics of rumor spreading," Annals of Optimization Theory and Practice, vol. 1, no. 3, pp. 45-54, 2018.

[7] L. Wang, J. X. Zhang, and J. H. Zhang, "Research on the dissemination of internet rumors with multiple spread sources and related guidance and control model," Information Science, vol. 11, pp. 115-120, 2020.

[8] M. D. Kermark and A. G. Mckendrick, "Contributions to the mathematical theory of epidemics," Proceedings of the Royal Society A: Mathematical, Physical and Engineering Sciences, vol. 115 , no. 5 , pp. $700-721,1927$.

[9] D. P. Maki and M. Thompson, Mathematical Models and Applications: With Emphasis on the Social, Life, and Management Sciences, Prentice Hall, Hoboken, NJ, USA, 1973.

[10] Y. Zan, J. Wu, P. Li, and Q. Yu, "SICR rumor spreading model in complex networks: counterattack and self-resistance," Physica A: Statistical Mechanics and its Applications, vol. 405, pp. 159-170, 2014.
[11] S. Dong, Y.-B. Deng, and Y.-C. Huang, "SEIR model of rumor spreading in online social network with varying total population size," Communications in Theoretical Physics, vol. 68, no. 4, pp. 545-552, 2017.

[12] L. H. Zhu, X. Zhou, and Y. M. Li, "Global dynamics analysis and control of a rumor spreading model in online social networks," Physica A: Statistical Mechanics and its Applications, vol. 526, pp. 1-15, 2019.

[13] Y. Zhang and J. Zhu, "Stability analysis of I2S2R rumor spreading model in complex networks," Physica A: Statistical Mechanics and its Applications, vol. 503, pp. 862-881, 2018.

[14] P. Jia, C. Wang, G. Zhang, and J. Ma, "A rumor spreading model based on two propagation channels in social networks," Physica A: Statistical Mechanics and its Applications, vol. 524, pp. 342-353, 2019.

[15] J. Wang, H. Jiang, T. Ma, and C. Hu, "Global dynamics of the multi-lingual SIR rumor spreading model with cross-transmitted mechanism," Chaos, Solitons \& Fractals, vol. 126, pp. 148-157, 2019.

[16] L. Huo and X. Chen, "Dynamical analysis of a stochastic rumor-spreading model with holling II functional response function and time delay," Advances in Difference Equations, vol. 651, pp. 1-21, 2020.

[17] L. Qiu, W. Jia, W. Niu et al., "SIR-IM: SIR rumor spreading model with influence mechanism in social networks," Soft Computing, pp. 1-10, 2020.

[18] R. Zhang and D. Li, "Identifying influential rumor spreader in social network," Discrete Dynamics in Nature and Society, vol. 2019, Article ID 8938195, 10 pages, 2019.

[19] D. H. Zanette, "Dynamics of rumor propagation on smallworld networks," Physical Review. E, Statistical, Nonlinear, and Soft Matter Physics, vol. 65, no. 4, Article ID 041908, 2002.

[20] Y. Moreno, M. Nekovee, and A. F. Pacheco, "Dynamics of rumor spreading in complex networks," Physical Review. E, Statistical, Nonlinear, and Soft Matter Physics, vol. 69, no. 6, Article ID 066130, 2004.

[21] M. Nekovee, Y. Moreno, G. Bianconi, and M. Marsili, “Theory of rumor spreading in complex social networks," Physica A: Statistical Mechanics and its Applications, vol. 374, no. 1, pp. 457-470, 2007.

[22] L. H. Zhu, W. S. Liu, and Z. D. Zhang, "Delay differential equations modeling of rumor propagation in both homogeneous and heterogeneous networks with a forced silence function," Applied Mathematics and Computation, vol. 370, pp. 1-22, 2020.

[23] W. Yan, Q. Feng, P. C. Jian, and P. N. Ye, "Spreading dynamics of a $2 \mathrm{SIH} 2 \mathrm{R}$ rumor spreading model in the homogeneous network," Complexity, vol. 2021, Article ID 6693334, 9 pages, 2021.

[24] L. Lü, D. B. Chen, and T. Zhou, "The small world yields the most effective information spreading," New Journal of Physics, vol. 13, no. 12, 2011.

[25] J. Wang and Y. Q. Wang, "SIR rumor spreading model with network medium in complex social networks," Chinese Journal of Physics, vol. 53, no. 1, pp. 1-21, 2015.

[26] L. Zhu and Y. Wang, "Rumor spreading model with noise interference in complex social networks," Physica A: Statistical Mechanics and its Applications, vol. 469, pp. 750-760, 2017.

[27] J. M. Zhang and L. Q. Qiu, “A rumour propagation model with suspicion mechanism in social networks," Software Guide, vol. 20, no. 4, pp. 123-128, 2021.

[28] L. Zhao, Q. Wang, J. Cheng, Y. Chen, J. Wang, and W. Huang, "Rumor spreading model with consideration of forgetting 
mechanism: a case of online blogging live journal," Physica A: Statistical Mechanics and its Applications, vol. 390, no. 13, pp. 2619-2625, 2011.

[29] K. Afassinou, "Analysis of the impact of education rate on the rumor spreading mechanism," Physica A: Statistical Mechanics and its Applications, vol. 414, pp. 43-52, 2014.

[30] X. Liang and Y. Wan, "The rumor spreading model with the interference of media and its control strategy," Journal of Nanjing University of Posts and Telecommunications (Natural Science Edition), vol. 37, no. 1, pp. 120-126, 2017.

[31] P. Jiang and X. Yan, "Stability analysis and control models for rumor spreading in online social networks," International Journal of Modern Physics C, vol. 28, no. 5, pp. 1-22, 2017.

[32] W. Liu, X. Wu, W. Yang, X. Zhu, and S. Zhong, "Modeling cyber rumor spreading over mobile social networks: a compartment approach," Applied Mathematics and Computation, vol. 343, pp. 214-229, 2019.

[33] S. Dong, F.-H. Fan, and Y.-C. Huang, "Studies on the population dynamics of a rumor-spreading model in online social networks," Physica A: Statistical Mechanics and its Applications, vol. 492, pp. 10-20, 2018.

[34] Q. Y. Gu, C. H. Ju, and F. G. Bao, "Simulation research on dynamic evolution of rumor spreading user group on mobile social networks via integration improvement of SIR model," Information Science, vol. 37, no. 10, pp. 67-74, 2019.

[35] E. B. Amine, G. Rachid, B. R. Soukaina, R. Mostafa, and E. Adil, "A discrete mathematical modeling and optimal control of the rumor propagation in online social network," Discrete Dynamics in Nature and Society, vol. 2020, Article ID 4386476, 12 pages, 2020. 